\title{
Feasibility study on EEG driven robotic system to realize efficient stroke rehabilitation
}

Conference or Workshop Item

Accepted Version

Nagai, K., Goto, T., Shimizu, T., Dobashi, H., Ito, K., Hayashi, Y., Loureiro, R. C. V., Nasuto, S. and Harwin, W. S. (2015) Feasibility study on EEG driven robotic system to realize efficient stroke rehabilitation. In: ICORR 2015: 11th International Conference on Rehabilitation Robotics, 11-14 August 2015, Singapore, pp. 199-204. doi:

https://doi.org/10.1109/ICORR.2015.7281199 Available at http://centaur.reading.ac.uk/40679/

It is advisable to refer to the publisher's version if you intend to cite from the work. See Guidance on citing.

To link to this article DOI: http://dx.doi.org/10.1109/ICORR.2015.7281199 
including copyright law. Copyright and IPR is retained by the creators or other copyright holders. Terms and conditions for use of this material are defined in the End User Agreement.

\section{www.reading.ac.uk/centaur}

\section{CentAUR}

Central Archive at the University of Reading

Reading's research outputs online 


\title{
Feasibility Study on EEG Driven Robotic System to Realize Efficient Stroke Rehabilitation
}

\author{
Kiyoshi Nagai*, Takaaki Goto*, Takuya Shimizu*, Hiroki Dobashi*, Koji Ito*, \\ Yoshikatsu Hayashi ${ }^{\dagger}$, Rui C. V. Loureiro ${ }^{\ddagger}$, Slawomir J. Nasuto ${ }^{\dagger}$ and William S. Harwin ${ }^{\dagger}$ \\ * College of Science and Engineering, Ritsumeikan University, Kusatsu, Shiga, 525-8577, Japan \\ Email: \{nagai@se,rr0023sv@ed,rr0023ep@ed, dobashi@fc, koji-ito@fc\}.ritsumei.ac.jp \\ $\dagger$ School of Systems Engineering, University of Reading, Reading RG6 6AY, UK \\ Email: \{y.hayashi, s.j.nasuto, w.s.harwin\}@ reading.ac.uk \\ $\ddagger$ Aspire Centre for Rehabilitation Engineering and Assistive Technology, \\ University College London, Brockley Hill, Stanmore, Middlesex, London HA7 4LP, UK \\ Email: r.loureiro@ucl.ac.uk
}

\begin{abstract}
We aim to develop an efficient robotic system for stroke rehabilitation, in which a robotic arm moves the hemiplegic upper limb when the patient tries to move it. In order to achieve this goal we have considered a method to detect the motion intention of the patient using EEG (Electroencephalogram), and have designed a rehabilitation robot based on a Redundant Drive Method. In this paper, we propose an EEG driven rehabilitation robot system and present initial results evaluating the feasibility of the proposed system.
\end{abstract}

\section{INTRODUCTION}

Several rehabilitation robots have been developed for stroke rehabilitation over the last decade and tested in clinical settings. The MIT-MANUS has been developed to deliver planar motions in upper limb rehabilitation to people following a stroke [1], and commercialized as a therapy system by Interactive Motion Technologies [2]. Another example is the GENTLE/s which has been developed as a robotic sensorimotor system for delivery of $3 \mathrm{D}$ motions in upper limb rehabilitation after a stroke [3].

A recent study suggests that pre-programmed exercises should be replaced by Repetitive Facilitation Exercises (RFEs), in which the patient's intention to move the hemiplegic upper limb or finger is followed by realization of the movement and multiple sensory stimulations [4]. For example, Event-Related Desynchronization (ERD) modulated by Functional Electrical Stimulation (FES) has been used on stroke patients with severe hemiparesis of lower limbs with promising results [5].

In order to assist people suffering from a stroke, we are developing an efficient rehabilitation robot system, in which a robotic arm moves the hemiplegic upper limb when the patient tries to move it. For this purpose, the system must be able to detect the motion intention. In order to make the system portable for use during the acute phase of recovery and subsequent chronic phases of rehabilitation, we propose to use EEG (Electroencephalogram) to detect the motion intention [6], instead of MRI (Magnetic Resonance Imaging) or NIRS (Near Infrared Spectroscopy). Other BCI methods have been reviewed in [7]. It is also required that the robotic system is able to provide compliant motions during rehabilitation. For this purpose, we proposed the use of the

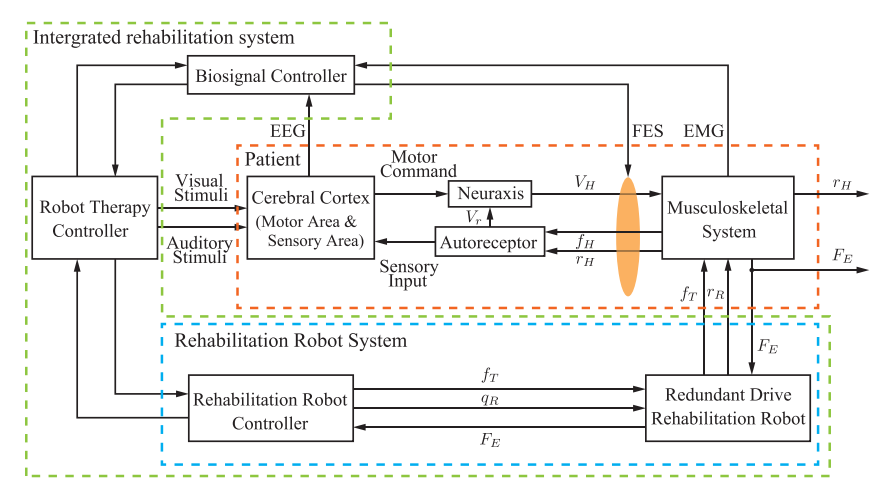

Fig. 1. EEG Driven Rehabilitation Robot System

Redundant Drive Method (RDM) in the design of the new rehabilitation robot [8]. In this literature, however, the total configuration of the proposed system and the feasibility of desired functions of elemental techniques are not discussed.

In this paper, we propose an EEG driven robotic system for stroke rehabilitation, and discuss the feasibility of the proposed system. The total configuration of the proposed system and the required component techniques are described in Section II. The EEG based system to detect motion intention is discussed in Section III. The prototype robotic arm based on the RDM is investigated in Section IV. The findings obtained in this paper are summarized in Section V.

\section{Basic Concept of Proposed Robotic System}

\section{A. Overview of the Proposed Robotic System}

We propose an integrated rehabilitation system for stroke rehabilitation as shown in Fig.1. This subsection explains the configuration of the integrated system and the roles of its three important controllers namely the robot therapy controller, the biosignal controller, and the robot controller.

The role of the biosignal controller is to detect useful information from EEG and EMG (Electromyography) measured from the patient and to manage Functional Electrical Stimulation (FES) given to the patient. The biosignal controller sends the detected information to the robot therapy controller and receives the command signal from it to drive FES. On the other hand, the role of the robot controller is 
to receive the position and the external force data of the rehabilitation robot, and to drive the robot with a tapping force mechanism considering RFEs as mentioned above. The robot therapy controller manages both the biosignal controller and the robot controller in order to make the rehabilitation robot system efficient. It also manages the audio and visual information which are given to the patient during the delivery of the rehabilitation.

\section{B. BCI Method to Detect Motion Intention Using EEG}

One important task of the biosignal controller is to detect the motion intention prior to the real motion of the patient's upper limb. The detected motion intention can be used to give a trigger to the robot controller in order to move the robot so that his/her upper limb can be guided regardless of the paralysis.

In order to establish the online detection method of the motion intention, the detected ratio and detected time error are important in the two cases: if the system fails to detect the motion intention, it cannot move the upper limb, and if the system leads the false detection, it moves the upper limb without the patient's intention.

\section{Redundant Drive Robot to Realize Compliant Motion}

One of the important tasks of the rehabilitation robot system is to move a patient's upper limb compliantly according to the motion intention of the patient. At the same time, the patient's upper limb posture must be kept away from those which hurt the patient, and the excessive forces on the patient must be avoided in order to ensure patient's safety.

Therefore, the rehabilitation robot is required to produce compliant motions. In particular, compliant motions in a higher bandwidth should be realized to prevent bottomhitting of the patient's arm even when an external force exerted on the robot increases suddenly.

\section{Detection of Motion Intention}

\section{A. Representative Frequency Method}

ERD is the brain activity associated with motor activity often used for BCI. During ERD, EEG power measured on the scalp over the motor cortex decreases in the frequency range of $\alpha$ and $\beta$ frequencies during motor imagery or prior to the motion onset [9]. However, EEG is susceptible to noise. In our research, it is necessary to detect ERD from only single trial during which EEG is measured since ERD must be detected on-line without any training for the rehabilitation robot system. To solve these problems, we propose the Representative Frequency Method (RFM).

The outline of the proposed method is as follows:

Step 1: $\quad$ EEG is measured;

Step 2: The EEG data obtained in Step 1 is decomposed into frequency components as a power spectrum;



Fig. 2. Configuration of the experimental setup: (a) Dry electrodes, (b)Input box, (c) Multi-channel amplifier, (d) A/D converter, (e) Counter board, (f) Target PC, (g) Host PC, (h) Equipment for measurement of upper limb angle

Step 3-1: Each frequency power estimated in Step 2 is weighted by tuning parameters;

Step 3-2: The representative frequency is calculated using the weighted power spectra in Step 3-1;

Step 4: $\quad$ Presence of motion intention is estimated using the value of the representative frequency calculated in Step 3-2.

For details of each step, see [6]. This method has the following advantages: i) it is hardly affected by the influence of noise since the single value, the representative frequency, calculated from the information in the wide frequency range is used, and ii) it can deal with individual differences of the users since the tuning parameters are used.

\section{B. Experimental Setup and Procedure}

In the experiment, EEG and the spontaneous motion of the upper limb is measured simultaneously from seven healthy subjects, and the time lag between the estimate of the motion intention and the onset of the motion is measured.

The configuration of the experimental setup is shown in Fig.2. EEG is measured using active dry electrodes (g.SAHARA electrode, $7 \mathrm{~mm}$, g.tec) from seven scalp sites (international 10-20 system, F3, Fz, F4, C3, Cz, C4, Pz), the reference electrode is placed on the right ear (A2), and the ground is taken on the left ear (A1). The electrode positions are over the motor cortex area. EEG signals are transferred to the target $\mathrm{PC}$ using MATLAB via the multi-channel amplifier (AB-611J, Nihon Kohden). The target motion is flexion of the upper limb, and the onset of the motion is recognized by detecting the onset of the change of the elbow angle, $q$, by the encoder implemented in the experimental 1DOF arm. Sampling frequency is set to $1,000[\mathrm{~Hz}]$. EEG is filtered using a band-pass filter from $0.5[\mathrm{~Hz}]$ to $40[\mathrm{~Hz}]$ in the input box (g.SAHARAbox, g.tec). The combination of these dry electrodes, input box and amplifier has a problem in the lower frequency of the output. However, since the RFM is constructed to detect the change in the EEG signals. Therefore, the result obtained by the proposed method has substantially no problem.

The measurement for a single trial is performed in the following procedure:

1) The electrodes are placed on the seven scalp sites of the subject. 
2) The elbow of the subject is placed on the pad of the experimental arm, and the wrist is fixed on the arm with a band.

3) The subject performs flexion of the upper limb once during ten seconds.

4) Ten trials are defined as one set, and ten sets are performed.

5) If some sets are measured continuously, subjects take a break for a few minutes between sets.

Our research and the above experimental procedure are approved by the ethical committee of Ritsumeikan Univesity. After the measurement, the obtained EEG data are analyzed by the proposed method.

\section{Result of Representative Frequency Method}

Fig.3 shows the time histories of the representative frequency and the elbow angle of the upper limb, $q$, as a function of time from the electrode on F3. In this paper, the onset time of the motion is coincided with time $t=0$ [ms], and this is considered to be the onset time of the motion of the upper limb. We use the data included within four seconds prior to the motion onset time. The trial data are measured from seven healthy adult men. In this paper, the results of analysis are given from the data measured from one of the subjects. This is because his number of trial data is the largest of the seven subjects. In this paper, we detect motion intention by the following procedure. First, the time history of the representative frequency, $X_{G}$, in the period of time from $t-T_{x}$ to $t$, where $T_{x}(>0)$ is a constant time, is considered as shown in Fig.4. Second, the time such that $X_{G}$ takes the maximum value denoted by $X_{G a}$ in this time span is defined as $t_{a}$. Then, if the value of $X_{G}$ falls below $X_{G a}-S_{x}$, where $S_{x}$ is a constant magnitude of frequency, at time $t$, the time is defined as the detection time of motion intention denoted by $t_{b}$.

Fig. 5 shows the distribution of the detection time, $t_{b}$, and the drop ratio of the value of $X_{G}$ given by

$$
\alpha=\frac{X_{G b}-X_{G a}}{t_{b}-t_{a}},
$$

for all of the experimental trials. Here we set as $T_{x}=1,000$ [ms] and $S_{x}=1.5[\mathrm{~Hz}]$. In this paper, $S_{x}$ is set to a constant value while $S_{x}$ can be a tuning parameter in general. The average of $t_{b}$ is $-1,483$ [ms], and the standard deviation of $t_{b}$ is 723 [ms]. The histogram of $t_{b}$ is shown in Fig.6. From the figure, it can be seen that the motion intention is detected before the motion onset time in $79 \%$ of trials (before: 71 trials, after: 16 trials, non-detected: 3 trials). For introducing the proposed method into practical use, it is required to improve the detected ratio of the motor command generation and to make the errors in the detected times shorter.

\section{Compliant Motion Guidance}

\section{A. Proposed Mechanism of a Redundant Drive Robot}

In our proposed system, the robotic part that connects to the patient's upper arm incorporates a redundant drive robot (RDR). The structure of an RDR has the advantage to reduce

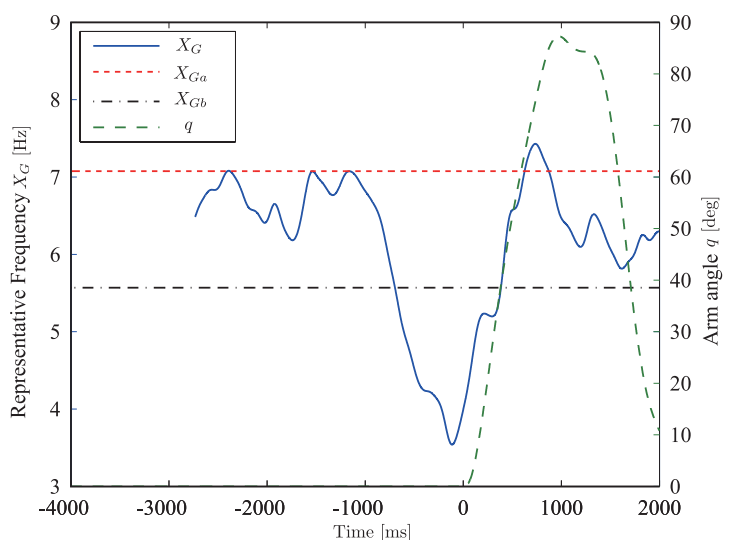

Fig. 3. Time histories of the representative frequency and the elbow angle of the upper limb as a function of time from the electrode on F3

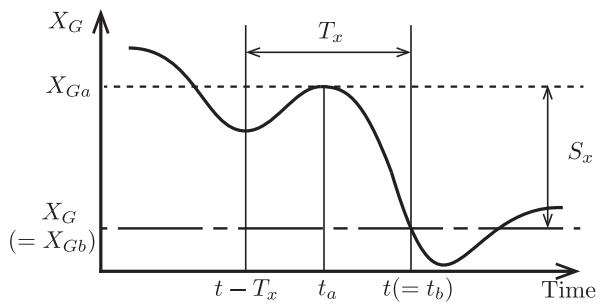

Fig. 4. Definition of the detection time of motion intention

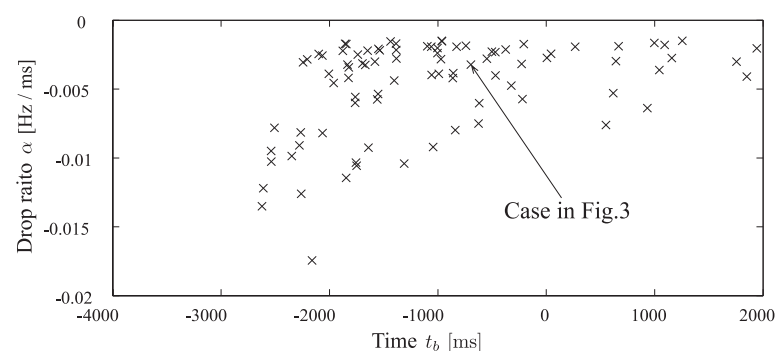

Fig. 5. Distribution of $\alpha$

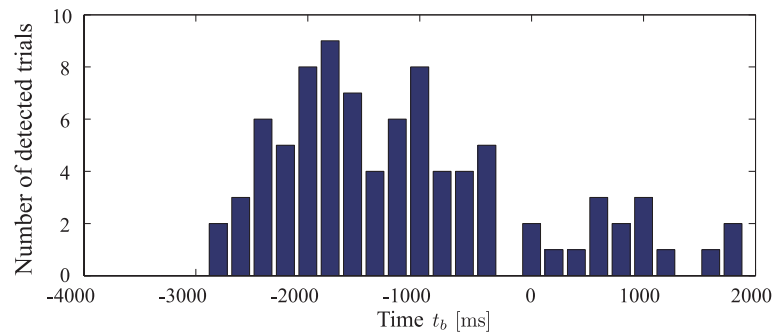

Fig. 6. Histogram of the detection time

the endpoint inertia in its motion direction, so the RDR can produce high frequency compliant motions. In this section, the proposed RDR mechanism is described.

Fig.7 shows the basic structure of the RDR. The RDR has two actuators connected in serial, and the driving forces are transmitted by wires. This manipulator has mainly two links. As shown in Fig.5, the second link with a small inertia is attached to the first link at the endpoint of the first link. The first link is driven by the sum of the outputs of the two actuators, and the second link is driven by the difference of those actuators. The kinematics and the statics of the RDR 


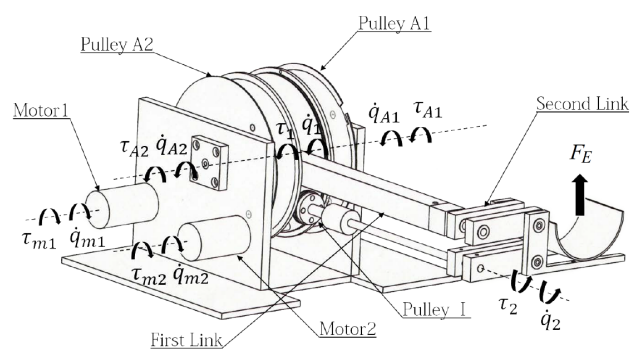

Fig. 7. Basic structure of the RDR

shown in Fig.7 are given by

$$
\begin{gathered}
{\left[\begin{array}{c}
\dot{r}_{1} \\
\dot{r}_{M D}
\end{array}\right]=\tilde{\boldsymbol{J} \dot{\boldsymbol{q}}}, \quad \tilde{\boldsymbol{J}}=\left[\begin{array}{c}
\tilde{\boldsymbol{J}}_{1} \\
\boldsymbol{J}_{M D}
\end{array}\right], \quad \tilde{\boldsymbol{J}}_{1}=\left[\begin{array}{ll}
J_{M D 1} & 0
\end{array}\right],} \\
\boldsymbol{\tau}=\tilde{\boldsymbol{J}}^{\mathrm{T}} F,
\end{gathered}
$$

where $\dot{\boldsymbol{q}}=\left[\begin{array}{ll}\dot{q}_{1} & \dot{q}_{2}\end{array}\right]^{\mathrm{T}}$ is the set of angular velocities of the links, $\tau=\left[\begin{array}{ll}\tau_{1} & \tau_{2}\end{array}\right]^{\mathrm{T}}$ is the set of driving torques of the links, $\tilde{\boldsymbol{J}}$ is the Jacobian matrix which relates angular velocities of the links and the endpoint velocities of the first link and the whole manipulator in its motion direction, $\boldsymbol{J}_{M D}$ is the Jacobian matrix which relates angular velocities of the actuators and the endpoint velocity in its motion direction, and $F$ is the endpoint force in its motion direction, respectively.

The equation of motion of the RDR in the $\boldsymbol{q}$-space is given as follows [8]:

$$
\boldsymbol{M} \ddot{\boldsymbol{q}}+\boldsymbol{g}(\boldsymbol{q})=\boldsymbol{\tau}+\boldsymbol{J}_{M D}^{\mathrm{T}} F_{E},
$$

where $\ddot{\boldsymbol{q}}=\left[\begin{array}{ll}\ddot{q}_{1} & \ddot{q}_{2}\end{array}\right]^{\mathrm{T}}$ is the set of angular accelerations of the links, $F_{E}$ is the external force applied at the endpoint of the second link in its motion direction, $\boldsymbol{M}$ is the inertia matrix of the manipulator in $\boldsymbol{q}$-space, and $\boldsymbol{g}(\boldsymbol{q})$ is the gravity force, respectively. Here it is assumed that the Coriolis force can be neglected since the RDR is intended to use for rehabilitation, and thus the motions of the manipulator should be slow.

The endpoint inertia of the manipulator in its motion direction $M_{M D}$ is given as follows [8]:

$$
\begin{aligned}
M_{M D} & =\left(\boldsymbol{J}_{M D} \boldsymbol{M}^{-1} \boldsymbol{J}_{M D}^{\mathrm{T}}\right)^{-1} \\
& =\left(M_{L 1}^{-1}+M_{L 2}^{-1}\right)^{-1}+\left(M_{1}^{-1}+M_{2}^{-1}\right)^{-1},
\end{aligned}
$$

where $M_{L 1}, M_{L 2}$ and $M_{1}, M_{2}$ are the inertias of the links and the actuators projected to the motion direction of the endpoint, respectively. From (5), it can be seen that both the influence of the inertias of the actuators and that of the inertia of each link can be reduced if either $M_{L 1}$ or $M_{L 2}$ and either $M_{1}$ or $M_{2}$ can be reduced. Therefore, the mechanism of the RDR has the advantage to reduce the endpoint inertia, and so the RDR can produce compliant motions in a higher frequency bandwidth.

\section{B. Control Scheme}

In this section, the impedance control method of the RDR is explained. The mechanical impedance is specified only at the endpoint in the case of ordinary impedance control [10]. However, in our control scheme [11], the mechanical impedances are specified not only at the endpoint of the whole manipulator but also at the tip of the macro part of the manipulator. In the impedance control of the RDR, the two desired mechanical impedances are specified to the endpoint of the whole manipulator, and the endpoint of the macro part, i.e., the first link of the manipulator, as follows:

$$
\widetilde{\boldsymbol{M}}_{d}\left[\begin{array}{c}
\ddot{r}_{1} \\
\ddot{r}_{M D}
\end{array}\right]+\widetilde{\boldsymbol{D}}_{d}\left[\begin{array}{c}
\dot{r}_{1 e} \\
\dot{r}_{M D e}
\end{array}\right]+\widetilde{\boldsymbol{K}}_{d}\left[\begin{array}{c}
r_{1 e} \\
r_{M D e}
\end{array}\right]=\left[\begin{array}{c}
F_{1 I} \\
F_{E}
\end{array}\right],
$$

where $F_{1 I}$ is the internal force in the mechanism caused by the external force applied at the endpoint of the manipulator. $r_{M D e}, \dot{r}_{M D e}$ and $\ddot{r}_{M D}$ are the deviation of the endpoint position of the whole manipulator, that of the velocity of the whole manipulator and the acceleration of the endpoint of the whole manipulator, respectively, $r_{1 e}, \dot{r}_{1 e}$ and $\ddot{r}_{1}$ are the deviation of the endpoint position of the first link, that of the velocity of the endpoint of the first link, the acceleration of the endpoint of the first link, respectively, $\widetilde{\boldsymbol{M}}_{d}, \widetilde{\boldsymbol{D}}_{d}$ and $\widetilde{\boldsymbol{K}}_{d}$ are the desired inertia matrix, the desired viscous coefficient matrix, and the desired stiffness matrix.

The control law to realize the desired impedance described by (6) is given by eliminating the acceleration term from (4) and (6).

$$
\begin{array}{r}
\boldsymbol{\tau}=\boldsymbol{M} \tilde{\boldsymbol{J}}^{-1} \widetilde{\boldsymbol{M}}_{d}^{-1}\left(\left[\begin{array}{c}
F_{1 I} \\
F_{E}
\end{array}\right]-\widetilde{\boldsymbol{D}}_{d}\left[\begin{array}{c}
\dot{r}_{1 e} \\
\dot{r}_{M D e}
\end{array}\right]\right. \\
\left.-\widetilde{\boldsymbol{K}}_{d}\left[\begin{array}{c}
r_{1 e} \\
r_{M D e}
\end{array}\right]\right)+\boldsymbol{h}-\boldsymbol{J}_{M D}^{\mathrm{T}} \boldsymbol{F}_{E}
\end{array}
$$

\section{Simulation}

In order to ensure safety of the patient, in this section, we confirm the validity of the control law and verify the effect of the compliant motion guidance by conducting a simulation in which we apply the control scheme in Section IV-B. In the control scheme, we apply the inertia projected from $\boldsymbol{q}$-space to the control-space as the desired inertia. The desired stiffness is determined from the demand motion, and the desired viscosity is determined so that the damping coefficient becomes 1 , as follows:

$$
\begin{aligned}
\widetilde{\boldsymbol{M}}_{d} & =\left[\begin{array}{cc}
M_{1 d} & 0 \\
0 & M_{M D d}
\end{array}\right]=\left[\begin{array}{cc}
0.259[\mathrm{~kg}] & 0 \\
0 & 0.115[\mathrm{~kg}]
\end{array}\right], \\
\widetilde{\boldsymbol{K}}_{d} & =\left[\begin{array}{cc}
K_{1 d} & 0 \\
0 & K_{M D d}
\end{array}\right]=\left[\begin{array}{cc}
1,000[\mathrm{~N} / \mathrm{m}] & 0 \\
0 & 1,000[\mathrm{~N} / \mathrm{m}]
\end{array}\right], \\
\widetilde{\boldsymbol{D}}_{d} & =\left[\begin{array}{cc}
D_{1 d} & 0 \\
0 & D_{M D d}
\end{array}\right]=\left[\begin{array}{cc}
2 \sqrt{M_{1 d} K_{1 d}} & 0 \\
0 & 2 \sqrt{M_{M D d} K_{M D d}}
\end{array}\right] .
\end{aligned}
$$

Figs. 8 and 9 show the simulation result of the endpoint positions of the manipulator and the detected external force. In these figures, the displacement of the second link is denoted by the time history of $r_{2}=r_{M D}-r_{1}$. In this simulation, the external force of $-20[\mathrm{~N}]$ is constantly applied at the endpoint of the second link in its motion direction from $t=0[\mathrm{~s}]$, which is equivalent to the weight of the upper arm of a human, and the impulsive external force of $-80[\mathrm{~N}]$ is applied at $t=8[\mathrm{~s}]$. The desired positions of the endpoints of the first link and the whole manipulator 


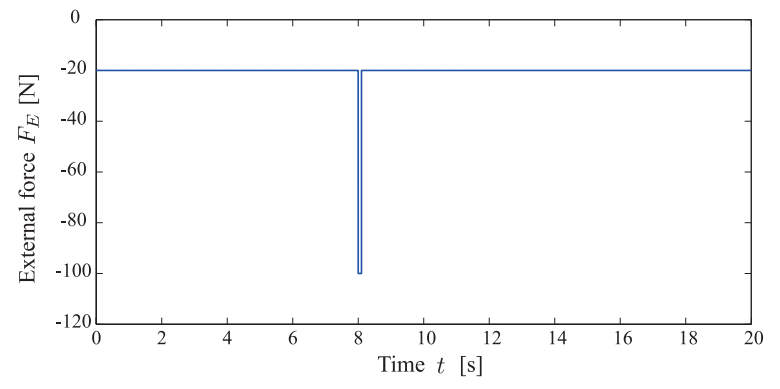

(a) External force

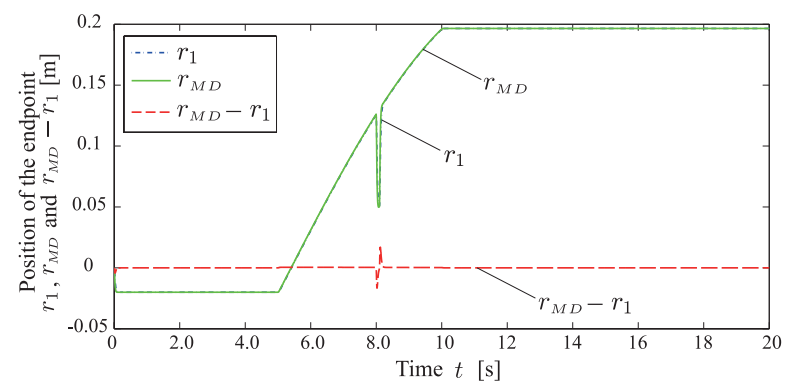

(b) Position of the endpoint of each link

Fig. 8. Relationship between position of the endpoint of each link and external force

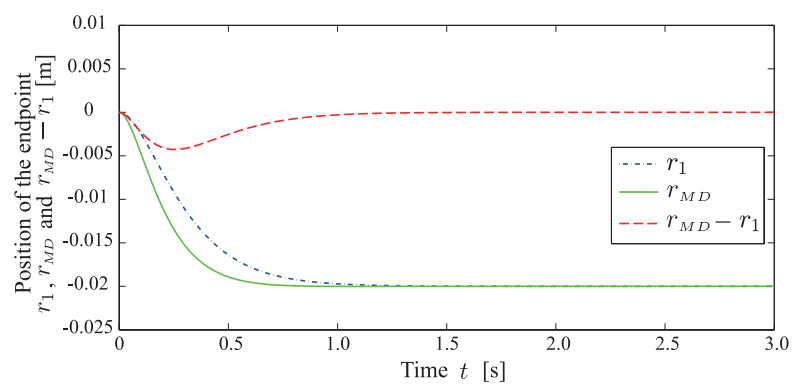

(a) Position of the endpoint of each link (0 $[\mathrm{s}] \leq t \leq 3[\mathrm{~s}])$

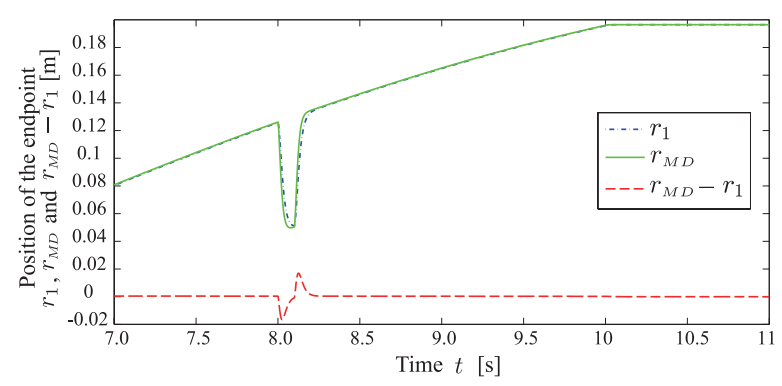

(b) Position of the endpoint of each link (7 [s] $\leq t \leq 11[\mathrm{~s}])$

Fig. 9. Effect of each ink

are given as the first link turns 60 degrees from $t=5[\mathrm{~s}]$ to $t=10[\mathrm{~s}]$.

Figs.9(a) and (b) show that as soon as the external force is exerted, $r_{2}$, the position of the endpoint of the second link, reacts firstly, and then $r_{1}$, the position of the endpoint of the first link, follows $r_{M D}$.

This is because the inertia of the second link is smaller than that of the first link, and thus the acceleration of the endpoint of the second link is larger than that of the first link. In other words, the endpoint of the second link moves in the

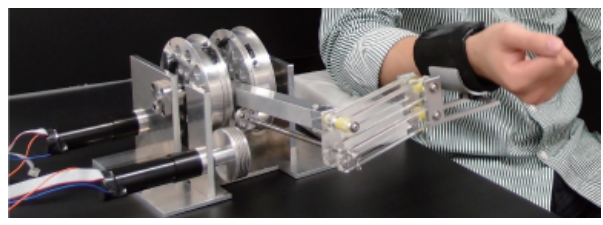

Fig. 10. Prototype of the RDR

higher frequency bandwidth, and the endpoint of the first link moves in the lower frequency bandwidth. The reason why each link works effectively at different bandwidth range. Considering that the mechanical motion range of the second link in the prototype is $-0.07[\mathrm{~m}] \leq r_{2} \leq 0.07[\mathrm{~m}]$, the simulation result shows that the prototype can move without bottom-hitting. From this simulation, when the control scheme in Section IV-B is applied, the RDR can achieve compliant motion guidance without bottom-hitting even if the external force is suddenly exerted to the RDR.

\section{Experiment}

In this section, the validity of the method we propose as compliant motion guidance is verified by experiments. In the experiment, we apply the control scheme described in Section IV-B to the the prototype of the RDR shown in Fig.10, and the parameter of the desired inertia matrix $\widetilde{\boldsymbol{M}}_{d}$, the desired viscous coefficient matrix $\widetilde{\boldsymbol{D}}_{d}$, and the desired stiffness matrix $\widetilde{\boldsymbol{K}}_{d}$, are set the same as those of the simulation.

The upper arm is attached on the endpoint of the whole manipulator, and then the prototype of the RDR turns 60 degrees from $t=5[\mathrm{~s}]$ to $t=10[\mathrm{~s}]$. About at $t=6[\mathrm{~s}]$, the wearer of the RDR applies a certain external force to the $\mathrm{RDR}$, and this force is detected by strain gauges attached to the endpoint of the second link.

The experimental result is shown in Figs.11 and 12 . Fig. 11 shows the time histories of the endpoint position of each link of the manipulator and the detected external force. Fig.12 shows a part of the time history of the endpoint position of each link of the manipulator. From Figs.12(a) and (b), it can be seen that as soon as the external force is exerted, similarly to the simulation, the position of the endpoint of the second link $r_{2}$, reacts firstly, and then the position of the endpoint of the first link $r_{1}$ follows $r_{M D}$.

This means that each link works effectively at the different bandwidth range. Also, from Fig.11 (a), it can be seen that the prototype can move without bottom-hitting, considering that the mechanical motion range of the second link in the prototype is $-0.07[\mathrm{~m}] \leq r_{2} \leq 0.07[\mathrm{~m}]$.

However, Fig.11 shows that the endpoint of each link does not move about at $t=6[\mathrm{~s}]$, i.e., the moment the external force is applied at the endpoint of the manipulator. The reason why the sliding occurs between pulley I and wire. This problem could be solved by installing a mechanism which prevents the sliding.

\section{CONCLUSION}

In this paper, we proposed an EEG driven rehabilitation robot system for stroke rehabilitation, and characterized 


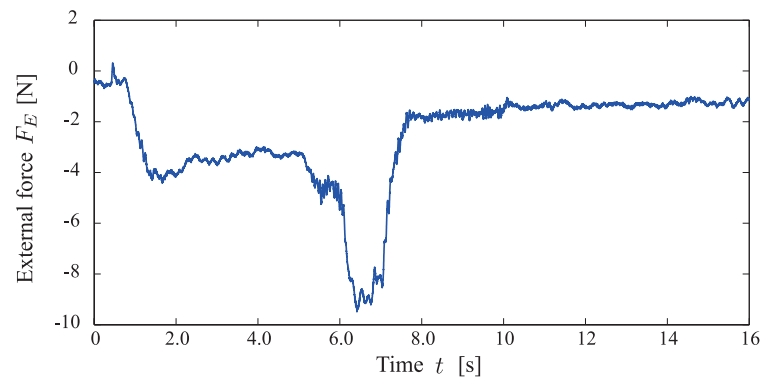

(a) External force

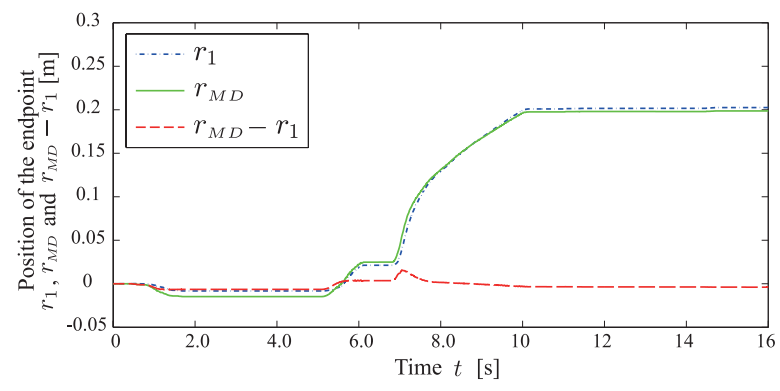

(b) Position of the endpoint of each link

Fig. 11. Relationship between position of the endpoint of each link and external force

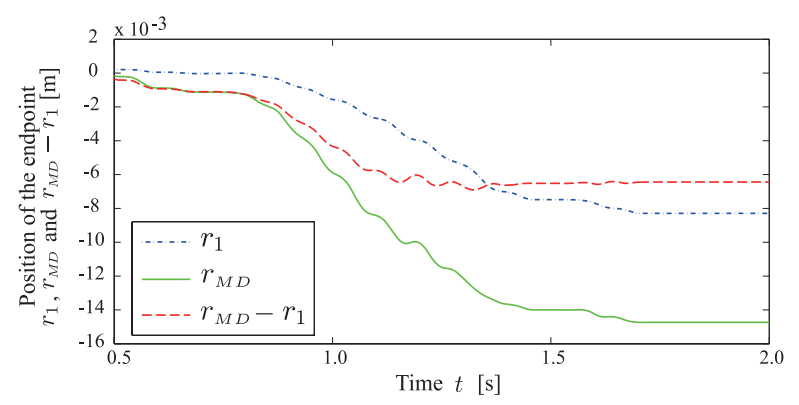

(a) Position of the endpoint of each link $(0.5[\mathrm{~s}] \leq t \leq 2[\mathrm{~s}])$

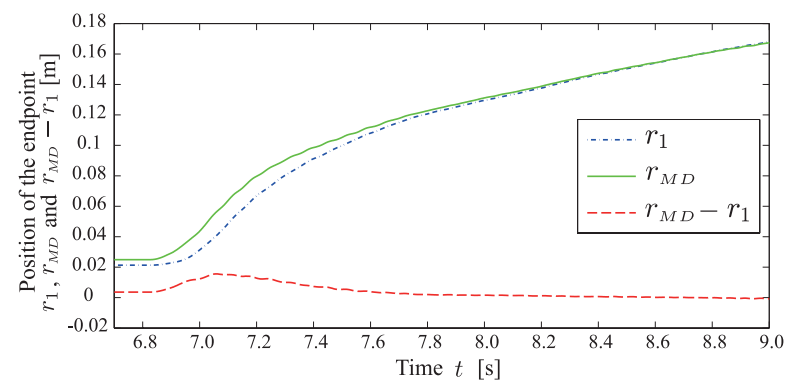

(b) Position of the endpoint of each link $(6.7[\mathrm{~s}] \leq t \leq 9[\mathrm{~s}])$

Fig. 12. Effect of each ink

experimentally its feasibility, as summarized below.

1) It is found that drops of the representative frequency of a certain subject can be detected prior to the start of the motion in $79 \%$ of trials by adopting the RFM. Then, the average of the detected times is $1,483[\mathrm{~ms}]$ prior to the onset of the motion, and the standard deviation of the detected times is $723[\mathrm{~ms}]$. For introducing the proposed method into practical use, it is required to improve the detected ratio of the motor command generation and to make the errors in the detected times shorter.
2) It is found that a prototype robotic arm which was designed based on the Redundant Drive Method (RDM) can move the human forearm with compliance not only at the start of the motion but also when an external force is applied at the human arm. The experimental results show that the RDM could be effectively used as a hardware component in the proposed rehabilitation system.

The next step of this research is to develop a rehabilitation robot prototype using the key technologies discussed in this paper, and to test it in clinical settings with both stroke and incomplete spinal cord injury patients at different phases of recovery.

\section{ACKNOWLEDGMENT}

This work was supported by JSPS KAKENHI Grant Number 24300206.

\section{REFERENCES}

[1] N. Hogan, H.I. Krebs, J. Charnnarong, P. Srikrishna and A. Sharon, MIT-MANUS: A Workstation for Manual Therapy and Training I, Proc. of the 1992 IEEE Int'l Workshop on Robot and Human Communication (RO-MAN '92), pp.161-165, 1992.

[2] Interactive Motion Technologies, http://interactive-motion.com/

[3] R. Loureiro, F. Amirabdollahian, M. Topping, B. Driessen and W. Harwin, Upper Limb Robot Mediated Stroke Therapy-GENTLE/s Approach, Autonomous Robots, Vol.15, No.1, pp.35-51, 2003.

[4] K. Kawahira, M. Shimodozono, S. Etho, K. Kamada, T. Noma and N. Tanaka, Effects of Intensive Repetition of a New Facilitation Technique on Motor Functional Recovery of the Hemiplegic Upper Limb and Hand, Brain Injury, Vol.24, No.10, pp.1202-1213, 2010.

[5] M. Takahashi, K. Takeda, Y. Otaka, R. Osu, T. Hanakawa, M. Gouko and K. Ito, Event Related Desynchronization-modulated Functional Electrical Stimulation System for Stroke Rehabilitation: A Feasibility Study, J. of NeuroEngineering and Rehabilitation, Vol.9, No.56, 2012.

[6] T. Shimizu, H. Dobashi, K. Ito and K. Nagai, Consideration on a New BCI Method Using EEG to Detect Motion Intention, Proc. of the 2014 IEEE/SICE Int'l Symp. on System Integration (SII '14), pp.785-789, 2014.

[7] K. K. Ang and C. Guan, Brain-Computer Interface in Stroke Rehabilitation, Journa of Computing Science and Engineering, Vol.7, No.2, pp.139-146, 2013.

[8] T. Goto, H. Dobashi and K. Nagai, Consideration on Mechanical Properties of a Redundant Drive Manipulator for Reducing the Endpoint Inertia, Proc. of the 2014 IEEE/SICE Int'1 Symp. on System Integration (SII '14), pp.767-772, 2014.

[9] G. Pfurtscheller, C. Brunner, A. Schlogl and F.H. Lopes da Silva: $\mathrm{Mu}$ rhythm (de)synchronization and EEG single-trial classification of different motor imagery tasks, NeuroImage, Vol.31, pp.153-159, 2006.

[10] N. Hogan, Impedance Control: An Approach to Manipulation: Part I-III, Trans. of ASME J. of Dynamic System, Measurement, and Control, Vol.107, pp.1-24, 1985.

[11] K. Nagai and T. Yoshikawa, Impedance Control of Redundant Macro Micro Manipulators, Proc. of the 1994 IEEE/RSJ Int'l Conf. on Intelligent Robots and Systems (IROS '94), Vol.2, pp.1438-1445, 1994. 